\title{
Parallelogram-shaped dielectric elastomer generators: Analytical model and experimental validation
}

Journal of Intelligent Material Systems and Structures 20I5, Vol. 26(6) 740-75। (C) The Author(s) 2014 Reprints and permissions: sagepub.co.uk/journalsPermissions.nav DOI: | 0.1 I 77// 045389X|456386 | jim.sagepub.com (SAGE

\author{
Giacomo Moretti, Marco Fontana and Rocco Vertechy
}

\begin{abstract}
Dielectric elastomers are smart materials that can be used to conceive solid-state electromechanical transducers such as actuators, sensors, and generators. Dielectric elastomer generators, in particular, are very promising for energy harvesting applications because they potentially feature large energy densities, good conversion efficiencies, good shock and corrosion resistance, and low cost. In this article, a novel concept of parallelogram-shaped dielectric elastomer generator is presented and analyzed. Parallelogram-shaped dielectric elastomer generators are rotary variable capacitance transducers, which are made by planar dielectric elastomer membranes that are covered with compliant electrodes and clamped along their perimeter to the links of a parallelogram four-bar mechanism. First, an analytical model for the electrohyperelastic response of the parallelogram-shaped dielectric elastomer generator is described and used to assess the maximum theoretical performances of the device. Then, an experimental case study with a parallelogram-shaped dielectric elastomer generator prototype featuring a natural rubber dielectric elastomer membrane and carbon conductive grease electrodes is presented. Simulation and experimental results demonstrate the practical feasibility of the parallelogram-shaped dielectric elastomer generator concept.
\end{abstract}

\section{Keywords}

Dielectric elastomer, generator, natural rubber, energy harvesting, rotary generator, four-bar mechanism

\section{Introduction}

Dielectric elastomers (DEs) are highly deformable nonconductive polymeric materials that can be employed to conceive variable capacitance transducers. DEs have been extensively investigated for sensing and actuation purposes (Carpi et al., 2008). In the last decade, several studies have demonstrated the possibility of using them for the implementation of generators, the so called dielectric elastomer generators (DEGs), which convert mechanical energy into direct electricity (Pelrine et al., 2001). One of the most promising applications for DEGs is in the field of wave energy harvesting (Chiba et al., 2008; Jean et al., 2012; Moretti et al., 2014; Vertechy et al., 2014a). In fact, in an application of this kind, DEGs may provide the following advantages: (1) light-weightiness, (2) good energy conversion efficiency that is rather independent of operation frequency, (3) easiness of manufacturing and assembly, (4) high shock and corrosion resistance, (5) low noise, and (6) low cost.

The energy harvesting performance of DEGs is highly dependent on their topology and size as well as on the electromechanical properties of the employed DE material (Koh et al., 2011). For DEGs made by acrylic elastomer membranes and undergoing equibiaxial deformations, theoretical energy densities over $1 \mathrm{~J} / \mathrm{g}$ have been predicted (Koh et al., 2011) and experimental energy densities up to $0.56 \mathrm{~J} / \mathrm{g}$ have been measured (Huang et al., 2013), although for a limited number of cycles only. Despite DEGs under equibiaxial loading are very useful for theoretical studies and for assessing the experimental performance of DE materials, their usage in realistic energy harvesting systems is rather impractical due to the difficulty of implementing kinematic linkages that make it possible to impose this kind of loading constraints on the DE membrane boundary. For practical energy harvesting applications, different DEG architectures have been proposed that feature conical shape (McKay et al., 2010; Wang et al., 2012; Zhu et al., 2011), inflatable diaphragms (Kaltseis et al., 2011; Rosati Papini et al.,

Scuola Superiore Sant'Anna, Pisa, Italy

\section{Corresponding author:}

Rocco Vertechy, PERCRO SEES, Scuola Superiore Sant'Anna, Piazza Dei Martiri Della Libertà 33, Pisa, Italy.

Email: r.vertechy@sssup.it 
2013), and lozenge shape (Moretti et al., 2013). The choice of the specific architecture depends on the application requirements such as type of mechanical input, force-stroke characteristic, and encumbrance.

This article introduces the parallelogram-shaped dielectric elastomer generator (PS-DEG) and investigates its energy harvesting performances via both theoretical arguments and experiments. Compared to existing topologies, the PS-DEG is the first direct-drive rotary DEG that makes it possible to produce electricity from the motion of a rocker arm without the need of any additional mechanical transmission.

In this article, sections "PS-DEG" and "Analytical model of PS-DEG operation and control" introduce an analytical electro-hyperelastic model that describes the electromechanical response of PS-DEGs and that can be used to study its energy conversion performances; section "Experimental characterization of a DE material: mechanical properties and electrical tests" presents the material properties of a reference DE material (OPPO Band Red 8012) that has been considered for the development of a PS-DEG prototype; section "Analysis of the energy harvesting performances of the PS-DEG" presents the procedure for the optimal selection of the design parameters of PS-DEGs subjected to operational constraints; section "Experimental validation" presents an experimental case study, which validates the feasibility of the proposed PS-DEG architecture as well as the adequacy of the considered electromechanical model.

\section{PS-DEG}

This section describes the operating principle of the PSDEG and introduces an energy-based mathematical model that can be used for the representation of its electromechanical response. The PS-DEG is a planar variable capacitor, which consists in a parallelogram mechanism (namely, a four-bar mechanism with opposite sides of equal length) that accommodates in its interior an active deformable membrane (ADM) made by one or more DE films alternatively stacked with compliant electrode layers. DE films are required to have (1) large deformability, (2) large dielectric strength, (3) large dielectric constant, (4) low viscosity, and (5) low electrical conductivity. DE films can be made by silicone elastomer, acrylic elastomer or natural rubber (Carpi et al., 2008; Koh et al., 2011). Compliant electrodes are required to (1) be conductive; (2) remain bonded to the DE material during the cyclical deformation; and (3) be highly compliant, in order to provide the system with minimal extra-stiffness. Electrodes can be made by carbon powder or grease, conductive rubber, or metallic thin films (Rosset and Shea, 2013).

With reference to Figure 1(a), the PS-DEG has one fixed link, with length $l_{1}$ lying along the $\xi$ direction,

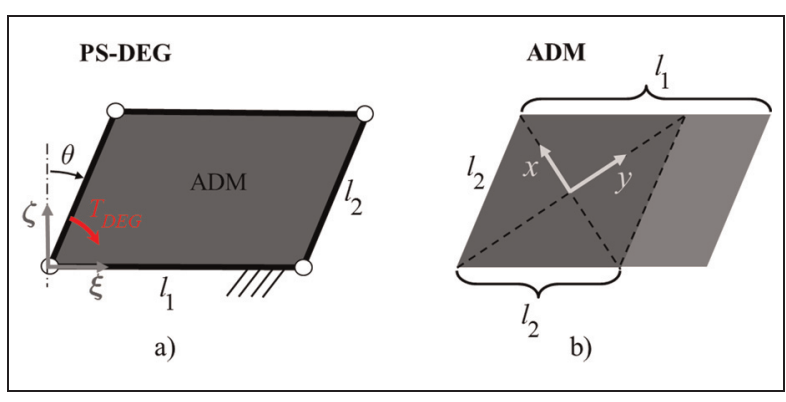

Figure I. (a) Schematic of a parallelogram-shaped dielectric elastomer generator and (b) scheme of the active deformable membrane that shows the principal strain directions $x-y$.

and one moving link, with length $l_{2}$ and being inclined by the angle $\theta$ with respect to the $\zeta$-axis; $l_{1}$ and $l_{2}$ are also the lengths of the ADM edges that, together with $\theta$, define the ADM area $A\left(A=l_{1} l_{2} \cos \theta\right)$. Assuming the DE material as being incompressible, as $\theta$ increases, the ADM area $A$ reduces and its thickness $t$ increases. Since the capacitance of a planar condenser is given by $C=\varepsilon A / t$, where $\varepsilon$ is the absolute permittivity of the dielectric medium, the PS-DEG capacitance decreases monotonically with increasing $\theta$.

Functionally, the PS-DEG can be used as a rotary generator with reciprocating motion to convert into electricity the mechanical work spent in a cycle by a torque applied to one of its oscillating links. The torque output, $T_{D E G}$, of the PS-DEG depends on the deformation and electrical states of the ADM and, thus, is function of the angular position, $\theta$, of the parallelogram mechanism and of the electric field, $E$, acting across the DE material.

To always be in a state of tension, the ADM is required to be mounted on the links of the mechanism with a certain amount of pre-stretch along the two principal directions of strain (see Figure 1(b)) that lie along the bisectors of the parallelogram angles. The amount of pre-stretch needs to be referred to a specific configuration identified by angle $\theta_{p}$. Here, the prestretches $\lambda_{1 p}$ and $\lambda_{1 p}$ are referred to the configuration $\theta_{p}=0$. Note that this configuration may not always be included in the angular operating range of the examined PS-DEG; however, it is always possible to virtually refer the pre-stretches to this angular position of the parallelogram.

With the abovementioned assumption, the stretch field within the ADM results as

$$
\lambda_{1}=\lambda_{1 p} \sqrt{1-\sin \theta}, \quad \lambda_{2}=\lambda_{2 p} \sqrt{1+\sin \theta}
$$

where $\lambda_{1}$ and $\lambda_{2}$ are the first (along $x$-direction) and second (along $y$-direction) principal stretches (see Figure 1(b)). Due to DE material incompressibility, the third principal stretch acting in the thickness direction follows as 


$$
\lambda_{3}=\left(\lambda_{1} \lambda_{2}\right)^{-1}
$$

The mathematical description of the electromechanical response of PS-DEGs can be obtained via an energy balance principle. The state of the generator is described by means of two independent variables. The geometric configuration is represented by $\theta$ and the electrical state of activation is described by the value of the electric field $E$ in the DE membrane. The charge $Q$ residing on the electrodes or the electric potential difference $V$ acting between the DEG terminals could also be chosen as alternatives in place of $E$.

The energy balance for a DEG can be expressed in differential form as it follows

$$
d U_{e}+d U_{m}=d W_{e}+d W_{m}
$$

The addenda appearing in the balance (3) represent, respectively:

- Electrostatic potential energy, $U_{e}$, that is stored in the DEG and given by

$$
U_{e}=\frac{\varepsilon \Omega E^{2}}{2}
$$

where $\Omega$ is the total volume of DE material.

- Elastic potential energy, $U_{m}$, that describes the mechanical state of tension of the DE membrane. Similar to other rubber-like materials, DEs can be considered as hyper-elastic solids admitting a strain-energy function $\Psi$ that governs their mechanical stress-strain response (Holzapfel, 2000). A review of the principal hyper-elastic models for rubber-like materials is presented by Steinmann et al. (2012). For PS-DEGs, the elastic potential energy assumes the following form that only depends on the variable $\theta$

$$
U_{m}=\Omega \Psi(\theta)
$$

- Electrical work, $W_{e}$, that is performed on the DEG by the external electric circuit, namely

$$
d W_{e}=V d Q
$$

Using $\theta$ and $E$ as state variables, and considering that the DEG capacitance is

$$
C=\frac{Q}{V}=\frac{\varepsilon l_{1}^{2} l_{2}^{2} \cos ^{2} \theta}{\Omega}
$$

the differential of the electrical work results as

$$
d W_{e}=\varepsilon \Omega E d E-\varepsilon \Omega E^{2} \tan \theta d \theta
$$

- Mechanical work, $W_{m}$, that is performed by the external torque applied to the parallelogram mechanism. Let $T_{D E G}$ be the torque provided by the DEG, which has same module and opposite sign of the externally applied torque. Then, for an infinitesimal rotation of the PS-DEG

$$
d W_{m}=-T_{D E G} d \theta
$$

In the considered energy balance (3), the following contributions have been neglected: (1) gravitational potential energy that is related to the orientation in space of the PS-DEG, (2) inertial terms that are related to the kinetic energy of the PS-DEG components, (3) visco-elasto-plastic losses that are due to the mechanical hysteresis of the DE material, and (4) electrical losses due to leakage currents through the DE material and to the resistivity of the deformable electrodes.

The combination of equation (3) with equations (4), (5), (8), and (9) yields an analytical expression for the torque provided by the PS-DEG. In particular, $T_{D E G}$ can be split into two addenda: an electrostatic contribution and an elastic mechanical term, namely

$$
\begin{aligned}
& T_{D E G}=T_{D E G, e}+T_{D E G, m}, \\
& T_{D E G, e}=-\varepsilon \Omega E^{2} \tan \theta, \\
& T_{D E G, m}=-\Omega \frac{d \Psi}{d \theta}
\end{aligned}
$$

The knowledge of the torque characteristic of the PS-DEG is important in order to assess its influence on the dynamic response of the external source from which mechanical energy is harvested. If required by the application, the mechanical component, $T_{D E G, m}$, can be compensated or corrected by means of passive mechanical components (for instance, linear or torsional springs) that can be properly mounted between the links of the parallelogram mechanism (Vertechy et al., 2010).

The amount of electrical energy that can be produced or spent for an infinitesimal displacement $d \theta$ of the PS-DEG is

$$
d U_{e}-d W_{e}=\varepsilon \Omega E^{2} \tan \theta d \theta
$$

When this expression is positive, electricity is generated. This happens when $\theta d \theta>0$ and $E \neq 0$. In this article, it is assumed that electric charges are present on the PS-DEG electrodes only during one half of the oscillation cycle, which corresponds to when the device capacitance decreases. In this phase, the applied external torque performs mechanical work against both the mechanical stresses of the membrane, which are due to DE material elasticity, and the electrically induced stresses, which are caused by the electrostatic attraction of the DEG electrodes. During the other phases, when $\theta d \theta \leq 0$ and $E=0$, the work performed by the applied external torque is balanced only by the variation of the elastic energy that is stored in the DE material. 


\section{Analytical model of PS-DEG operation and control}

In this section, the electromechanical limits for PSDEG operation are described and modeled analytically. The identification of the failure modes for a DEG provides important indications for generator operation and control.

DEGs perform a cyclical sequence of electromechanical transformations that is referred to as energy conversion cycle (ECC). ECCs can be represented on twoaxis diagrams by referring to a couple of independent state variables.

In scientific literature, ECCs are usually represented on the $\theta-T_{D E G}$ or the $Q-V$ planes (Koh et al., 2011). In this article, the $Q-V$ plane is used.

The range of admissible physical states for the PSDEG is bounded by a series of failure conditions (Koh et al., 2011) and operative constraints. On the $Q-V$ plane, these conditions are curves and enclose an operational surface, whose area is equal to the maximal energy $\left(E_{n}\right)$ that can be converted in a cycle by the PSDEG.

In the following, the fundamental practical constraints for the PS-DEG are described and modeled analytically. In order to simplify the mathematical analysis, the dielectric constant of the DE material is taken as independent of the stretch.

The combination of equations (1) and (7) provides a relation between the stretch field of the material and the state variables $Q$ and $V$, namely

$$
\begin{aligned}
& \lambda_{1}=\lambda_{1 p} \sqrt{1-\sqrt{1-\frac{\Omega}{\varepsilon l_{1}^{2} l_{2}^{2}} \frac{Q}{V}}}, \\
& \lambda_{2}=\lambda_{2 p} \sqrt{1+\sqrt{1-\frac{\Omega}{\varepsilon l_{1}^{2} l_{2}^{2}} \frac{Q}{V}}}
\end{aligned}
$$

Equations (12) indicate that iso-stretch transformations (i.e. transformations that occur with the parallelogram mechanism in a fixed position) are straight lines passing through the origin of the $Q-V$ plane.

As regards the DE stress field, electric activation is responsible for an electrostatically induced stress $\sigma_{e m}=\varepsilon E^{2}=Q V / \Omega$ that acts along the first and second principal directions of the DE membrane (Pelrine et al., 2001). Thus, the total principal stresses in the plane of the DE membrane follow as

$$
\begin{aligned}
& \sigma_{1}=\lambda_{1} \frac{\partial \Psi\left(\lambda_{1}, \lambda_{2}\right)}{\partial \lambda_{1}}-\sigma_{e m}, \\
& \sigma_{2}=\lambda_{2} \frac{\partial \Psi\left(\lambda_{1}, \lambda_{2}\right)}{\partial \lambda_{2}}-\sigma_{e m}
\end{aligned}
$$

Using the abovementioned equations, the limit curves for PS-DEG operation are obtained as follows.
Geometric constraint. In equations (12), the condition of the positive square root argument yields the limit curve

$$
V=\frac{\Omega Q}{\left(\varepsilon l_{1}^{2} l_{2}^{2}\right)}
$$

This condition physically means that the DEG capacitance reaches its maximum value, $C_{\max }=\varepsilon l_{1}^{2} l_{2}^{2} / \Omega$, when $\theta=0$.

Electrical breakdown condition. During PS-DEG operation, the electric field acting across the active membrane must be lower than the dielectric strength, $E_{B D}$, of the considered DE material. Previous works (Koh et al., 2011; Tröls et al., 2013) have employed either constant values for $E_{B D}$ or analytical relations expressing it as a function of DE membrane stretches.

Here, a constant $E_{B D}$ is assumed. However, based on experimental observations (Vertechy et al., 2014b), according to which the dielectric strength of certain DE materials may experience a drastic irreversible reduction in the case of excessive area expansion, the electrical breakdown condition takes the usual form $E \leq E_{B D}$ with the further constraint $\lambda_{1} \lambda_{2} \leq \Gamma$. Such conditions yield to the following limit curves

$$
\begin{gathered}
V Q=\varepsilon \Omega E_{B D}^{2} \\
V=\frac{\Omega \lambda_{1 p}^{2} \lambda_{2 p}^{2}}{\varepsilon l_{1}^{2} l_{2}^{2} \Gamma^{2}} Q
\end{gathered}
$$

On the $Q-V$ plane, the electrical breakdown curve (15) is a hyperbola, while constraint (16) represents a straight line.

Angular range constraints. For PS-DEGs operating with a fixed stroke, the endpoints of the angular operational range may be imposed, namely $\theta \leq \theta_{\max }$ and $\theta$ $\geq \theta_{\text {min }}$. These constrains may represent mechanical end-stops or other external limiting positions. Using equations (1) and (12), the resulting limit curves are

$$
\begin{aligned}
& V=\frac{\Omega}{\varepsilon\left(l_{1} l_{2} \cos \theta_{\text {max }}\right)^{2}} Q, \\
& V=\frac{\Omega}{\varepsilon\left(l_{1} l_{2} \cos \theta_{\text {min }}\right)^{2}} Q
\end{aligned}
$$

which are iso-capacitance curves consisting in straight lines passing through the origin of the $Q-V$ plane.

Mechanical rupture condition. The mechanical rupture of the DE material poses a limitation on the maximum principal stretch that can be borne by the membrane. The simplest fracture criterion is the Kawabata's condition (Hamdi et al., 2006), which asserts that rupture in the $i$-th principal direction does not occur if $\lambda_{i}<\lambda_{\max }$ (for $i=1,2,3$ ), where $\lambda_{\max }$ is a constant only dependent on the considered elastomeric material.

Since the DE membrane is pre-stretched in the plane of the DEG electrodes, the stretch in thickness 
direction, $\lambda_{3}$, is always lower than 1 . It is then sufficient to impose $\lambda_{1}<\lambda_{\max }$ and $\lambda_{2}<\lambda_{\max }$. Using equation (12), the resulting limit curves are

$$
\begin{gathered}
V=\frac{\Omega /\left(\varepsilon l_{1}^{2} l_{2}^{2}\right) Q}{1-\left(1-\lambda_{\max }^{2} / \lambda_{1 p}^{2}\right)^{2}}, \\
V=\frac{\Omega /\left(\varepsilon l_{1}^{2} l_{2}^{2}\right) Q}{1-\left(1-\lambda_{\max }^{2} / \lambda_{2 p}^{2}\right)^{2}}
\end{gathered}
$$

that represent straight lines crossing the origin of the $Q$ $V$ plane.

As a matter of fact, more sophisticated criteria exist in the literature for representing the mechanical rupture of elastomers (Hamdi et al., 2006), and thus for DEs. Nonetheless, the combination of the constraint $\lambda_{i}<\lambda_{\max }$ with the one described by $\lambda_{1} \lambda_{2} \leq \Gamma$ (which has been introduced as part of the electrical breakdown condition) provides a conservative limitation that can protect the employed DE material from mechanical rupture.

Loss-of-tension (or buckling) condition. For a correct operation, the principal stresses within the DE material must be positive. Otherwise, the DE membrane may wrinkle and the efficacy of the transduction process could be compromised. The loss-of-tension conditions for the principal directions yield to the following limit curves

$$
\lambda_{1} \frac{\partial \Psi\left(\lambda_{1}, \lambda_{2}\right)}{\partial \lambda_{1}}=\frac{Q V}{\Omega}, \quad \lambda_{2} \frac{\partial \Psi\left(\lambda_{1}, \lambda_{2}\right)}{\partial \lambda_{2}}=\frac{Q V}{\Omega}
$$

which highlight that the loss-of-tension limit is the only condition that depends on the hyper-elastic constitutive model chosen for the considered DE material.

In order to generalize the discussion and to adapt it to different choices of PS-DEG size and proportions, the following dimensionless charge and voltage variables

$$
Q^{*}=\frac{Q}{\varepsilon E_{B D} l_{1} l_{2}}, V^{*}=\frac{l_{1} l_{2}}{\Omega E_{B D}} V
$$

are introduced, and conditions (14) to (19) are reformulated by means of them.

With reference to the new variables, the mentioned constraints can be expressed in the following dimensionless form:

Dimensionless geometric constraint

$$
V^{*}=Q^{*}
$$

Dimensionless electrical breakdown condition

$$
Q^{*} V^{*}=1, V^{*}=\frac{\lambda_{1 p}^{2} \lambda_{2 p}^{2}}{\Gamma^{2}} Q^{*}
$$

Dimensionless angular range constraints

$$
V^{*}=\frac{Q^{*}}{\left(\cos \theta_{\max }\right)^{2}}, \quad V^{*}=\frac{Q^{*}}{\left(\cos \theta_{\min }\right)^{2}}
$$

Dimensionless mechanical rupture condition

$$
\begin{aligned}
V^{*} & =\frac{Q^{*}}{1-\left(1-\lambda_{\max }^{2} / \lambda_{1 p}^{2}\right)^{2}}, \\
V^{*} & =\frac{Q^{*}}{1-\left(1-\lambda_{\max }^{2} / \lambda_{2 p}^{2}\right)^{2}}
\end{aligned}
$$

Dimensionless buckling (loss-of-tension) condition

$$
\lambda_{1} \frac{\partial \Psi}{\partial \lambda_{1}}=\varepsilon E_{B D}^{2} Q^{*} V^{*}, \lambda_{2} \frac{\partial \Psi}{\partial \lambda_{2}}=\varepsilon E_{B D}^{2} Q^{*} V^{*}
$$

These equations make it possible to compare PSDEGs with different geometries but exploiting the same DE material. In fact, even if dimensionless variables are used, equation (25) presents a dependence on the electromechanical properties of the specific DE material considered for the application.

The surface enclosed by ECCs on the dimensionless $Q^{*}-V^{*}$ plane represents the dimensionless energy that can be converted by the PS-DEG in a cycle

$$
E_{n}^{*}=\frac{E_{n}}{\varepsilon E_{B D}^{2} \Omega}
$$

\section{Experimental characterization of a DE material: Mechanical properties and electrical tests}

The experimental case study described in the following sections employs a natural rubber DE (OPPO Band Red 8012). To determine the hyper-elastic strain-energy function, $\Psi\left(\lambda_{1}, \lambda_{2}\right)$, of the considered material, three cyclic tensile tests on pure-shear DE membrane specimens (Holzapfel, 2000) with different transversal prestretches, $\lambda_{2 p}$ (along the second principal direction), have been performed on a custom made tensile stage. Detailed explanation of experimental testing procedures and obtained results is provided by Vertechy et al. (2014b). The first specimen features initial (i.e. in its undeformed state) longitudinal length, transversal length and thickness equaling 15,150 , and $0.175 \mathrm{~mm}$, and a transversal pre-stretch $\lambda_{2 p}=1$. The second specimen features initial longitudinal length, transversal length and thickness equaling 15,105 , and $0.175 \mathrm{~mm}$, and a transversal pre-stretch $\lambda_{2 p}=2$. The third specimen features initial longitudinal length, transversal length and thickness equaling 15, 70, and $0.175 \mathrm{~mm}$, and a transversal pre-stretch $\lambda_{2 p}=3$. Specimens are tested in the longitudinal (first principal) direction with 


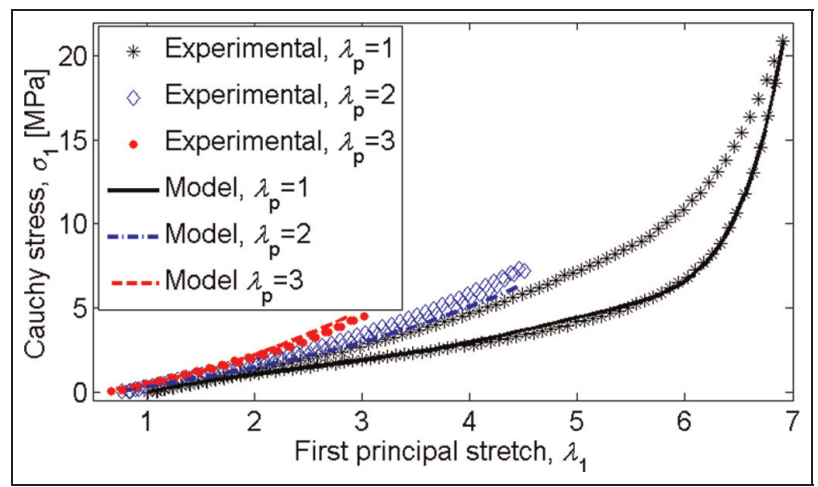

Figure 2. Stress-strain curves for the OPPO Band Red 8012: markers represent experimental data; lines represent the best fits (relative to the unloading curves only) with the same hyperelastic model.

cycles performed at a constant strain rate of $2 \mathrm{~s}^{-1}$ and up to a maximum first principal stretch of $\lambda_{1}=7$ for the first specimen $\left(\lambda_{2 p}=1\right), \lambda_{1}=4.5$ for the second specimen $\left(\lambda_{2 p}=2\right)$, and $\lambda_{1}=3$ for the third specimen $\left(\lambda_{2 p}=3\right)$. These maximum first principal stretches have been determined via the limiting conditions $\lambda_{\max }=7$ and $\lambda_{1} \lambda_{2} \leq \Gamma$ with $\Gamma=9$, which respectively prevents mechanical rupture and dielectric strength reduction in the considered natural rubber material (Vertechy et al., 2014b). To remove any stress-softening effects, before testing, all the three specimens have been subjected to five preconditioning cycles performed at a strain rate of $0.05 \mathrm{~s}^{-1}$ and up to the maximum first principal stretch. The resulting stabilized stress-strain curves are reported in Figure 2, which gives the longitudinal (first principal) Cauchy stress, $\sigma_{1}$, as a function of the longitudinal (first principal) stretch, $\lambda_{1}$, and of the transversal (second principal) pre-stretch, $\lambda_{2 p}$. As shown, the stabilized response of the considered DE material exhibits a significant hysteresis that is due to both viscosity and plasticity (Vertechy et al., 2014b). Specifically, Figure 2 shows that all pure-shear specimens exhibit larger stresses when the deformation is increasing (loading) than when the deformation is decreasing (unloading).

As regards electrical parameters, as described by Vertechy at al. (2014b), the limiting breakdown strength is chosen to be $E_{B D}=0.5 \times 80 \mathrm{MV} / \mathrm{m}$ (where $80 \mathrm{MV} / \mathrm{m}$ is the lower breakdown strength measured with rigid metal electrodes and 0.5 is a safety factor that accounts for a decrease in the dielectric strength that occurs when using carbon conductive grease electrodes), while the relative dielectric constant is considered in the range $\varepsilon_{r, \text { min }}=1.6$ and $\varepsilon_{r, \text { max }}=2.4$ (the maximum and minimum values being measured for unstretched and equi-biaxially stretched specimens with $\lambda_{1}=\lambda_{2}=3$, see Figure 3).

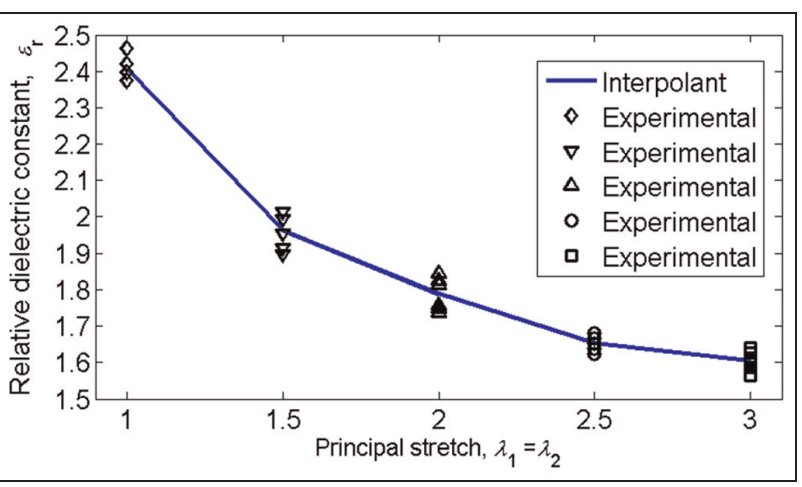

Figure 3. Dielectric constant as a function of equi-biaxial stretch for the OPPO Band Red 8012 .

\section{Fitting of the stress-strain experimental results}

The experimental data presented in Figure 2 have been used to determine the form and the parameters of the hyper-elastic strain-energy function, $\Psi\left(\lambda_{1}, \lambda_{2}\right)$. In the analysis, the mechanical hysteresis exhibited by the considered DE material (see Figure 2) has been neglected. Specifically, hyper-elastic model fitting has been performed by considering the unloading curves only. This choice has been made since the most important electromechanical processes in DEGs occur during the unloading of the DE material (namely, during the reduction in area of the active membrane). Specifically, this choice over-estimates the onset of the loss-oftension condition, which insures the correct operation of the DEG throughout its full range of motion. The fitting has been performed by considering altogether the three unloading curves obtained on the pure-shear specimens with transversal pre-stretches equaling $\lambda_{2 p}=1, \lambda_{2 p}=2$, and $\lambda_{2 p}=3$.

The hyper-elastic model considered for the fitting is the general version of the Mooney-Rivlin strain-energy function (Beda, 2007), that is

$$
\Psi=\sum_{i=1}^{n} C_{i 0}\left(I_{1}-3\right)^{i}+\sum_{j=1}^{m} C_{0 j}\left(I_{2}-3\right)^{j}
$$

where $I_{1}$ and $I_{2}$ are the first and second invariants of deformation, namely

$$
\begin{aligned}
& I_{1}=\lambda_{1}^{2}+\lambda_{2}^{2}+\left(\lambda_{1} \lambda_{2}\right)^{-2} \text { and } \\
& I_{1}=\lambda_{1}^{-2}+\lambda_{2}^{-2}+\left(\lambda_{1} \lambda_{2}\right)^{2}
\end{aligned}
$$

This strain-energy function has been chosen since the more traditional hyper-elastic models (Gent, Ogden, Mooney-Rivlin, Yeoh) produced poor fitting results on the considered experimental data, principally because of the strong dependence of the stress on the transversal pre-stretch. With relation (27), good fitting results have 
Table I. Experimental parameters of the hyper-elastic model.

\begin{tabular}{lccccc}
\hline$i, j$ & 1 & 2 & 3 & 4 & 5 \\
\hline$C_{i 0}(\mathrm{~Pa})$ & $1.46 \mathrm{e} 5$ & $-7.25 \mathrm{e} 3$ & $3.49 \mathrm{e} 2$ & -8.47 & $8.07 \mathrm{e}-2$ \\
$C_{0 j}(\mathrm{~Pa})$ & $2.26 \mathrm{e} 4$ & & & \\
\hline
\end{tabular}

Table 2. Physical properties for the reference natural rubber elastomer.

Reference elastomer commercial name

Relative dielectric permittivity, $\varepsilon_{r}$ Dielectric strength, $E_{B D}(\mathrm{MV} / \mathrm{m})$ Hyper-elastic model parameters Limit stretch value, $\lambda_{\max }$

OPPO Band
Red 8012 (Latex)
I.6-2.4
40
see Table I
7

been obtained for $n=5$ and $m=1$. The resulting fitting coefficients are reported in Table 1. Comparison between experimental and fitted stress-strain curves is shown in Figure 2.

\section{Analysis of the energy harvesting performances of the PS-DEG}

In this section, the proposed analytical model of the PS-DEG (see section "Analytical model of PS-DEG operation and control") is employed to optimize generator performance according to given operational constraints. The formalism of ECCs is used and the $Q^{*}-V^{*}$ dimensionless diagrams are adopted for graphical representation purposes. The DE material considered for the optimization is the OPPO Band Red 8012 whose main relevant physical properties, evaluated as described in section "Experimental characterization of a DE material: mechanical properties and electrical tests," are summarized in Table 2.

Since the presented model is valid for constant values of the dielectric permittivity, the calculations are made for both the maximum and minimum values of the measured relative dielectric constant $\varepsilon_{r}$ (namely, for both $\varepsilon_{r}=1.6$ and $\varepsilon_{r}=2.4$ ). The obtained results define a range within which the response of the experimental prototype is expected to lie. Figure 4 reports a graphical example of an ECC obtained for $\mathrm{DE}$ membrane pre-stretches equaling $\lambda_{l p}=8.5$ and $\lambda_{2 p}=1$, and by neglecting any particular constraint on the PS-DEG stroke. As shown, the use of different values for the relative dielectric constant only produces a slight variation in the shape of the loss-oftension curves.

The cycle reported in Figure 4 is composed by the following sequence of electromechanical transformations:

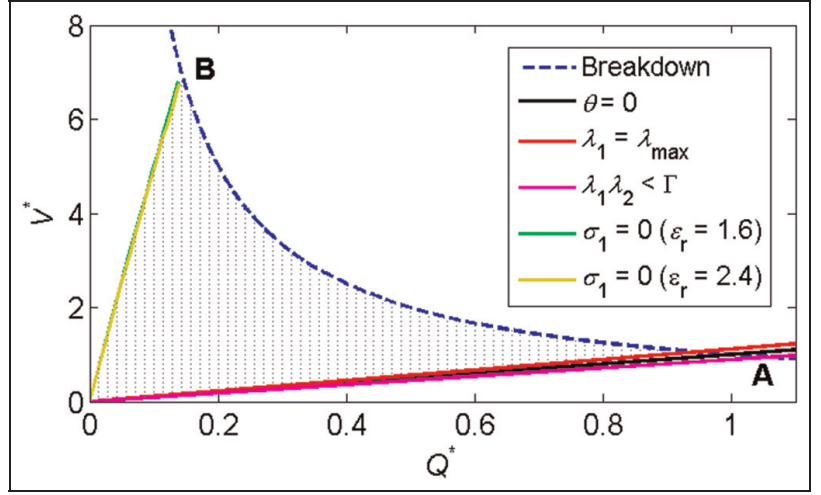

Figure 4. Example of energy conversion cycle on the dimensionless charge-voltage plane.

- Transformation $0-A$ represents the charging phase, which takes place at constant angular displacement. In this phase, the membrane is fixed in a configuration identified by the condition $\lambda_{1}=\lambda_{\max }$, which corresponds to a pitch angle $\theta=18.8^{\circ}$. Point $\mathrm{A}$ is encountered when the electric field inside the DE membrane reaches the value given by $E_{B D}$.

- Transformation $A-B$ takes place at constant electric field $E=E_{B D}$. In this phase, the angle $\theta$ increases and the PS-DEG capacitance decreases. To keep the electric field constant while the PS-DEG capacitance decreases, charge is progressively removed from the electrodes. Point B is found as the loss-of-tension curve is encountered (i.e. when $\sigma_{1}=0$ ).

- Transformation $B-0$ is featured by a constant value of stress (namely, $\sigma_{1}=0$ ). During this phase, the membrane is completely discharged. The final geometric configuration of the PSDEG is identified by the slope of the tangent to the loss-of-tension curve evaluated at the $Q^{*}-V^{*}$ plane origin, in this case corresponding to $\theta=82^{\circ}$.

After the conclusion of transformation $B-0$, the PSDEG is then brought back to its maximally expanded configuration. This is a purely mechanical transformation and it is not represented on the $Q^{*}-V^{*}$ diagram.

The dotted surface enclosed by the ECC is proportional to the energy converted per unit volume of DE. 


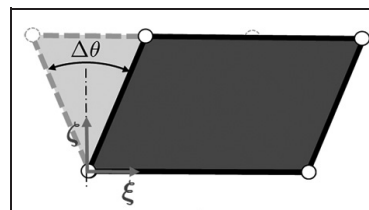

a)

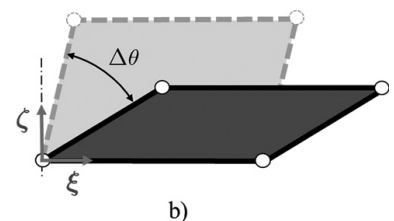

Figure 5. Extreme operational intervals for the parallelogramshaped dielectric elastomer generator (PS-DEG): (a) PS-DEG oscillating symmetrically around $\theta=0$ and (b) PS-DEG oscillating up to the end-stop position $\left(\theta_{\max }=\theta_{E S}\right)$.

For the example of Figure 4, the converted energy per unit of DE material volume results in 43 and $64 \mathrm{~kJ} / \mathrm{m}^{3}$ for $\varepsilon_{r}=1.6$ and $\varepsilon_{r}=2.4$, respectively.

\section{PS-DEG with stroke constraint}

In many practical cases, the angular stroke, $\Delta \theta$, of the PS-DEG may be imposed by the specific application. The required stroke can be implemented using different choices for the operational angular interval, $\left[\theta_{\min } ; \theta_{\max }\right]$. Since $\Delta \theta=\theta_{\max }-\theta_{\min }$, the operational range is uniquely identified by $\theta_{\min }$.

Assuming a stroke $\Delta \theta=40^{\circ}$, the maximal energy that can be converted by the PS-DEG can be calculated for different choices of $\theta_{\min }$, with the further constraint $\theta_{\max } \leq \theta_{E S}\left(\theta_{E S}=80^{\circ}\right)$ in order to avoid an excessive superposition of the links of the parallelogram mechanism. The considered range for $\theta_{\min }$ goes from $\theta_{\min }=-\Delta \theta / 2 \quad$ (Figure 5(a)) to $\theta_{\min }=\theta_{E S}-\Delta \theta$ (Figure 5(b)). If $\theta_{\min } \geq 0$, the generator performs one ECC per oscillation. If $\theta_{\min }<0$, configuration $\theta=0$ is included in the operational range of the DEG, and the generator performs two ECCs per oscillation. Figure 6 shows how the maximal energy density that can be converted during a full stroke of the PS-DEG varies as a function of $\theta_{\min }$, for the two different values of dielectric permittivity.

The following can be observed:

- Variations in $\theta_{\min }$ produce a difference of more than one order of magnitude on the energy that can be converted.

- The PS-DEG is more effective when operating away from the symmetric configuration. In fact, large values of $\theta$ guarantee larger variations of the parallelogram area, and thus of the PS-DEG capacitance, therefore allowing for larger energy conversion capabilities.

- Converted energies associated with different values of dielectric constants are scaled by a factor that is equal to the ratio of the dielectric constant magnitudes.

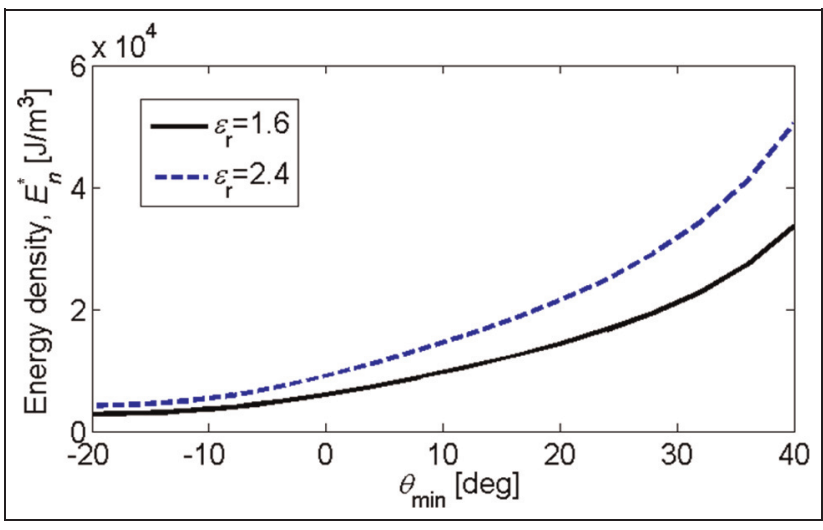

Figure 6. Energy density of the parallelogram-shaped dielectric elastomer generator (PS-DEG) as a function of the stroke starting angle, $\theta_{\min }$, for the imposed stroke $\Delta \theta=40^{\circ}$.

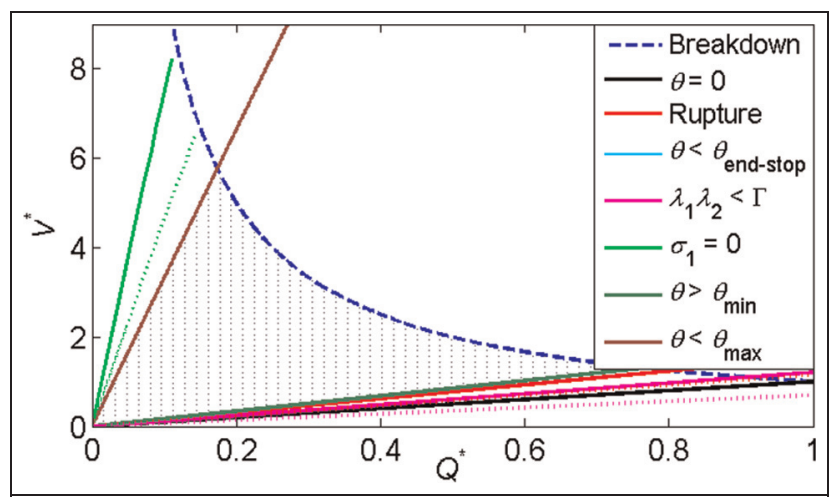

Figure 7. Energy conversion cycle of the parallelogram-shaped dielectric elastomer generator (PS-DEG) for $\theta_{\text {min }}=\theta_{E S}-\Delta \theta$ and for two different choices of the pre-stretches.

Figure 7 reports an example of ECC for the optimal case with $\varepsilon_{r}=1.6$ and $\theta_{\min }=40^{\circ}$, and for two distinct choices of DE membrane pre-stretches. Solid lines refer to $\lambda_{1 p}=11$ and $\lambda_{2 p}=0.9$; dashed lines refer to $\lambda_{1 p}=8.8$ and $\lambda_{2 p}=0.85$. The ECC is bounded by the limit curves representing the following conditions: (1) $\theta \geq \theta_{\min }$, (2) $E \leq E_{B D}$, and (3) $\theta \leq \theta_{\max }$. On the $Q^{*}-V^{*}$ plane, a variation of the pre-stretches does not produce a displacement of the curves. Variations of $\lambda_{1 p}$ and $\lambda_{2 p}$ only affect the curves that represent mechanical rupture, buckling, and $\lambda_{1} \lambda_{2}=\Gamma$ conditions. Consequently, for an adequate range of choices for $\lambda_{1 p}$ and $\lambda_{2 p}$, the ECC does not vary and the energy that can be converted is independent of the prestretches. Since, in dimensionless terms, a variation in the relative dielectric constant produces a variation in the buckling curve only, different values for $\varepsilon_{r}$ produce identical ECCs on the $Q^{*}-V^{*}$ plane.

Out of an appropriate range for $\lambda_{1 p}$ and $\lambda_{2 p}$, a dependence of the ECC on the pre-stretches occurs. In this 


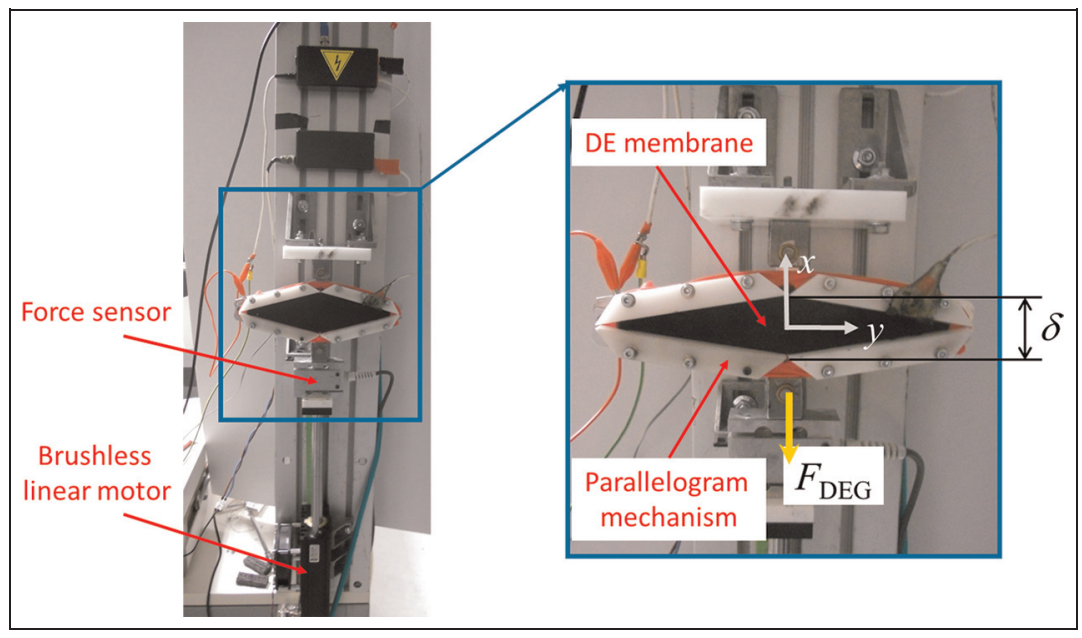

Figure 8. Experimental setup for testing a parallelogram-shaped dielectric elastomer generator (PS-DEG).

situation, in fact, low values of $\lambda_{1 p}$ and $\lambda_{2 p}$ provoke the loss-of-tension curve to reduce its slope and to pose a further limitation to the amount of energy that can be converted.

\section{Experimental validation}

A prototype of a PS-DEG generator has been built and a set of experimental tests has been conducted with the aim of validating the proposed generator concept along with its model. The prototype features a parallelogram mechanism with links having identical length $l_{1}=l_{2}=$

$120 \mathrm{~mm}$. The experimental setup is composed by the following mechanical equipment:

- An active membrane made by a single layer of natural rubber (Oppo Band Red 8012) with a thickness of $175 \mu \mathrm{m}$ (in the unstretched state) and two electrodes obtained with a carbon conductive grease (MG-Chemicals 846);

- A parallelogram mechanism whose links have been purposely designed to firmly connect the active membrane along its perimeter;

- A brushless linear motor (LinMot P01-37x120F/ 200x280-HP) equipped with a high-resolution linear encoder employed to impose the reciprocating motion to the PS-DEG;

- A single-axis load cell (DSEUROPE 535QD-A1) with a range of measurement of $\pm 250 \mathrm{~N}$;

and the following electrical equipment:

- A high-voltage power amplifier (Trek 10/10B$\mathrm{HS}$, with $\pm 10 \mathrm{kV}$ and $\pm 10 \mathrm{~mA}$ voltage and current ratings);

- A custom made electronic board equipped with a high-voltage switch;
- A high-voltage probe composed by a voltage divider to measure in real-time the electric potential applied to the active membrane;

- Driving and control electronics to regulate the PS-DEG motion and to acquire position, force and voltage signals.

The linear motor is employed to impose controlled displacements to the PS-DEG along the diagonal lying in the $x$-direction, with the length of this diagonal being indicated with $\delta$ (see Figure 8). The setup guarantees a force measurement accuracy and resolution better than $0.1 \mathrm{~N}$, position accuracy and resolution better than $0.1 \mathrm{~mm}$, and an acquisition rate up to $2 \mathrm{kHz}$. The charge and discharge of the active membrane can be synchronized with the movement of the linear motor in a way to accurately regulate the flows of electrical energy from and to the active membrane.

\section{Mechanical response validation}

A first set of measurements has been conducted with no electrical load in order to compare the experimental torque-angle characteristic of the PS-DEG with the theoretical response described by equation (10) using hyper-elastic model defined according to section "Experimental characterization of a DE material: mechanical properties and electrical tests."

Before installation, the natural rubber membrane has been subjected to the pre-stretches $\lambda_{1 p}=7.13$ and $\lambda_{2 p}=1.30$. The tests have been conducted by imposing saw-tooth position profiles with $\delta$ ranging between 30 and $165 \mathrm{~mm}$ (corresponding to an angular position $\theta$ ranging between $3^{\circ}$ and $80^{\circ}$ ) at a speed of nearly $30 \mathrm{~mm} / \mathrm{s}$, and by acquiring the force $F_{D E G}$ acting along the $x$-direction via the load cell. Upon measurements, force and position $\left(F_{D E G}\right.$ and $\delta$ ) measurements have been converted to the torque and angle $\left(T_{D E G}\right.$ and $\left.\theta\right)$ 
counterparts via the virtual work principle. Comparison of the experimental data with those predicted by the model is reported in Figure 9. As illustrated, the analytical model shows a good match with the experimental unloading curve, especially for the smaller values of $\theta$. For larger values of $\theta$, the analytical model tends to underestimate the force response of the PS-DEG, which makes the considered hyper-elastic strain-energy function more conservative for the forecast of the loss-of-tension condition.

\section{Generator model validation}

In order to assess the behavior of the system during generation cycles, the PS-DEG prototype has been subjected to a reciprocating motion similar to the one described above, but with $\theta$ ranging between $\theta_{\min }=2^{\circ}$ and $\theta_{\max }=75^{\circ}$, and the following electrical cycle at constant charge has been implemented:

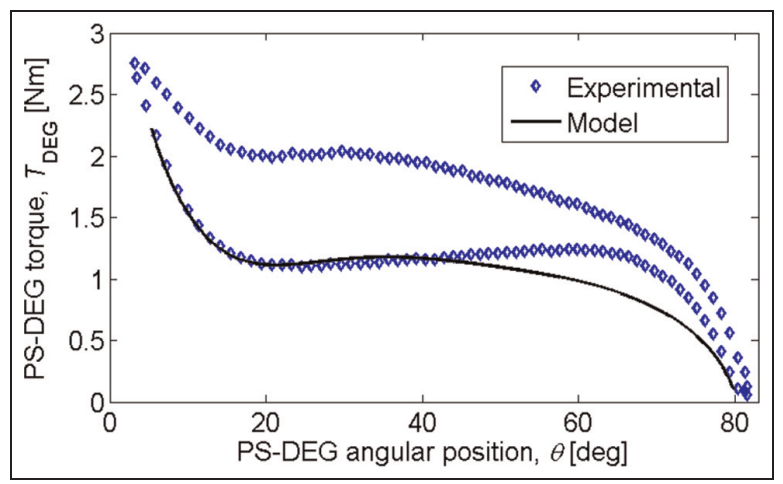

Figure 9. Comparison of experimental and theoretical torqueangle curves for the parallelogram-shaped dielectric elastomer generator.
S1: the PS-DEG is charged up to a voltage $V_{1}$ as soon as the mechanism reaches the minimum angular position $\left(\theta_{\min }\right)$ that corresponds to the maximum capacitance of the active membrane (a $10 \mathrm{~ms}$ charging time is used after which the PS-DEG electrodes are disconnected from the power supply via a highvoltage switch);

S2: PS-DEG electrodes are kept disconnected as the mechanism moves toward the maximum angular position $\left(\theta_{\max }\right)$ that corresponds to the minimum capacitance of the active membrane (in this phase, the electric potential between the PS-DEG electrodes increases, reaching the maximum value $V_{2}$ for $\left.\theta=\theta_{\max }\right)$;

S3: the PS-DEG is discharged to ground as soon as $\theta_{\text {max }}$ is reached;

S4: PS-DEG electrodes remain connected to ground as the mechanism moves back to the minimum angular position $\left(\theta_{\min }\right)$.

Experiments are conducted for two different values of charging voltage: $V_{1}=100 \mathrm{~V}$ and $V_{1}=617 \mathrm{~V}$. Results are illustrated in Figure 10 (left plot for $V_{1}=100 \mathrm{~V}$ and right plot for $V_{1}=617 \mathrm{~V}$ ), which reports the evolution of PS-DEG voltage with time. As expected, PS-DEG voltage increases as the angle $\theta$ increases and reaches its maximum value $V_{2}$ at $\theta=\theta_{\max }$ (specifically $V_{2}=750 \mathrm{~V}$ for $V_{1}=100 \mathrm{~V}$ and $V_{2}=4010 \mathrm{~V}$ for $V_{1}=617 \mathrm{~V}$ ). This demonstrates that the PS-DEG concept can be used for the implementation of practical generators.

For cycles at constant charge, the harvesting performances of DEGs can be represented by the voltage amplification ratio $\eta\left(\eta=V_{2} / V_{1}\right)$. For ideal PS-DEGs, which do not exhibit any electrical (and mechanical) dissipation, $\eta$ only depends on $\theta_{\min }$ and $\theta_{\max }$ via the theoretical formula

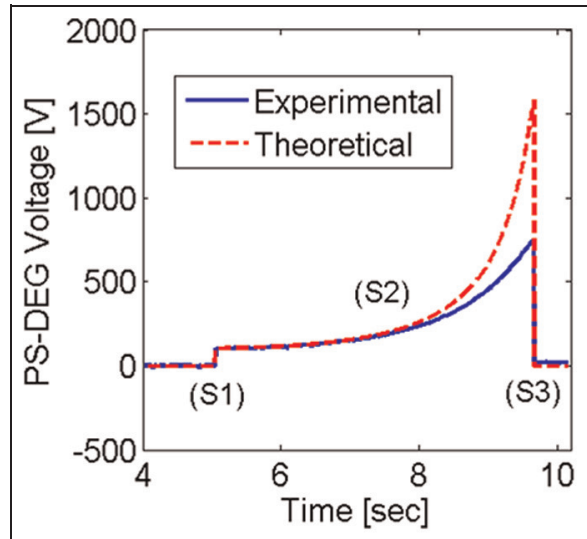

(a)

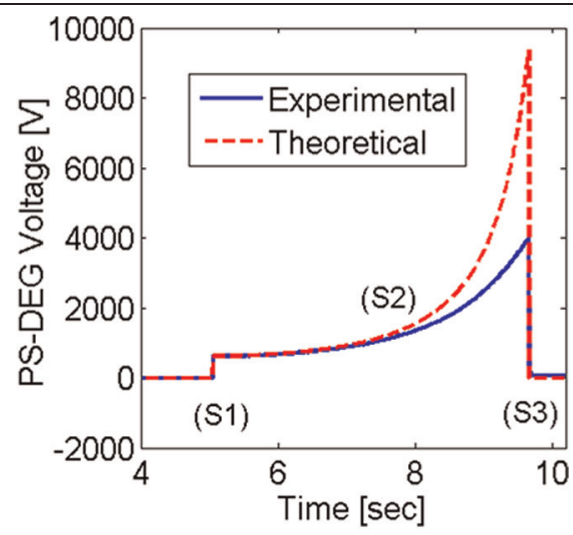

(b)

Figure 10. Time profile of electric potential difference between the parallelogram-shaped dielectric elastomer generator electrodes, for two different activation voltages: (a) $V_{1}=100 \mathrm{~V}$ and (b) $V_{1}=617 \mathrm{~V}$. 
Table 3. Voltage ratio: theoretical and experimental results comparison.

\begin{tabular}{llll}
\hline & Experiment, $V_{1}=100 \mathrm{~V}$ & Experiment, $V_{1}=617 \mathrm{~V}$ & Theoretical \\
\hline$\eta=V_{2} / V_{1}$ & 7.5 & 6.5 & 15.2 \\
\hline
\end{tabular}

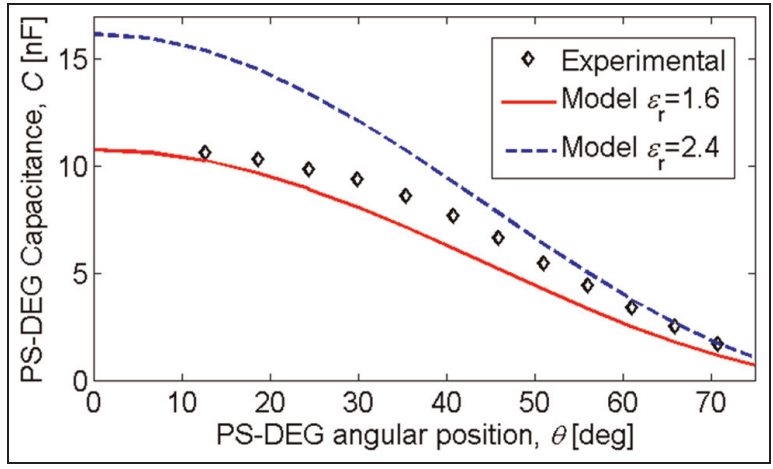

Figure I I. PS-DEG capacitance as a function of $\theta$. Theoretical (for $\varepsilon_{r}=1.6$ and $\varepsilon_{r}=2.4$ ) and experimental profiles.

$$
\eta=\frac{V_{2}}{V_{1}}=\frac{A_{1}^{2}}{A_{2}^{2}}
$$

where $A_{1}$ and $A_{2}$ are the areas of the parallelogram that correspond to the minimum and maximum angular positions, respectively.

Comparison between theoretically calculated and experimentally measured voltage ratios is shown in Table 3. For completeness, the theoretical evolution in time of the PS-DEG voltage that is predicted by equation (29) is also reported with dashed line in Figure 10.

The comparison highlights a noticeable disagreement between theoretical and experimental performances, which can be attributed in part to the variation of dielectric constant with stretch and in part to charge dispersions. As explained in section "Experimental characterization of a DE material: mechanical properties and electrical tests," the dielectric constant of the considered DE material reduces as soon as the stretch increases. In order to verify this effect on the PS-DEG, the capacitance of the prototype has been measured experimentally via a LCR bridge (Hameg Instruments LCR meter HM 8118) for different values of $\theta$. Results are reported in Figure 11, which compares them with the theoretical profiles of capacitance obtained using the minimum $\left(\varepsilon_{r}=1.6\right)$ and the maximum $\left(\varepsilon_{r}=2.4\right)$ dielectric constants. As shown, the experimental curve is included between the two theoretical profiles, confirming that the dielectric constant varies within the estimated range. Accordingly, the voltage ratio that could be expected from the capacitance measurements is $\eta=11$ (that is significantly smaller than the theoretical value $\eta=15.2$ ). The rest of the losses (from $\eta=11$ to $\eta=7.5$ or 6.5 ) are attributed to charge dispersion, both through the DE membrane and through air. In fact, for the same experimental conditions, larger losses occur for the largest electric potential differences. To diminish the dispersion losses through air, the electrode at higher electric potential should be insulated.

Based on voltage and capacitance measurements, the electrical energy scavenged in a cycle by the experimental PS-DEG prototype is calculated as $0.27 \mathrm{~mJ}$ for $V_{1}=100 \mathrm{~V}$ and $7.30 \mathrm{~mJ}$ for $V_{1}=617 \mathrm{~V}$. The corresponding energy densities are 1.0 and $26.8 \mathrm{~kJ} / \mathrm{m}^{3}$.

\section{Conclusion}

In this article, PS-DEGs are presented and investigated by means of theoretical models validated through experimental tests. PS-DEGs can effectively be employed as both rotary and linear transducers, and their mechanical response can vary largely depending on the chosen design parameters. The maximum theoretical performances of PS-DEGs have been evaluated considering the limits imposed by both material properties and operational constraints (such as fixed stroke and end-stops).

A practical case study is presented that considers a PS-DEG prototype based on a natural rubber dielectric elastomer membrane (OPPO Band Red 8012). Experimental tests validated both the PS-DEG concept and the developed theoretical models, demonstrating that the performance of PS-DEGs can be predicted quite accurately when the relevant electromechanical properties of the employed material are available.

In general, PS-DEGs present lower energy conversion capabilities with respect to other classes of DEGs that exploit uniform equi-biaxial deformations. Nonetheless, PS-DEGs are very interesting for practical applications since they are easy to implement and adapt in existing energy conversion devices, especially in those featuring links with oscillating rotational motion.

PS-DEGs based on soft elastomers (i.e. acrylics or silicones) manifest the tendency to wrinkle during operation. This problem is almost eliminated with the use of stiffer materials such as natural rubber, the operation of which is marginally influenced by the loss-of-tension condition.

The natural rubber specimen considered in this work exhibited a number of electrical issues. First, the measured relative dielectric constant is lower than that of acrylics and appears to decrease significantly with 
stretch. Second, a limiting value for the area expansion seems to exist beyond which the dielectric strength resistance is permanently degraded. Third, the electric conductivity of the material appears to be not negligible when it is operated at electric fields that are close to the breakdown strength.

\section{Declaration of conflicting interests}

The authors declared no potential conflicts of interest with respect to the research, authorship, and/or publication of this article.

\section{Funding}

The work presented in this article is developed in the context of the project PolyWEC (www.polywec.org), a FP7 FETEnergy project. The research leading to these results has received funding from the European Union Seventh Framework Programme (FP7/2007-2013) under grant agreement no. 309139.

\section{References}

Beda T (2007) Modeling hyperelastic behavior of rubber: a novel invariant-based and a review of constitutive models. Journal of Polymer Science Part B: Polymer Physics 45(13): 1713-1732.

Carpi F, De Rossi D, Kornbluh R, et al. (2008) Dielectric Elastomers as Electromechanical Transducers: Fundamentals, Materials, Devices, Models and Applications of an Emerging Electroactive Polymer Technology. Amsterdam: Elsevier Science Ltd.

Chiba S, Waki M, Kornbluh R, et al. (2008) Innovative power generators for energy harvesting using electroactive polymer artificial muscles. In: Proceedings of SPIE 6927: electroactive polymer actuators and devices (EAPAD), San Diego, CA, 9-13 March, pp. 692715-1-692715-9. Bellingham, WA: SPIE.

Hamdi A, Nait Abdelaziz M, Ait Hocine N, et al. (2006) A fracture criterion of rubber-like materials under plane stress conditions. Polymer Testing 25(8): 994-1005.

Holzapfel GA (2000) Nonlinear Solid Mechanics: A Continuum Approach for Engineering. New York: John Wiley \& Sons.

Huang J, Shian S, Suo Z, et al. (2013) Maximizing the energy density of dielectric elastomer generators using equi-biaxial loading. Advanced Functional Materials 23(40): 5056-5061.

Jean P, Wattez A, Ardoise G, et al. (2012) Standing wave tube electro active polymer wave energy converter. In: Proceedings of SPIE 8340: electroactive polymer actuators and devices (EAPAD), San Diego, CA, 11-15 March, pp. 83400C-1-83400C-21. Bellingham, WA: SPIE.

Kaltseis R, Keplinger C, Baumgartner R, et al. (2011) Method for measuring energy generation and efficiency of dielectric elastomer generators. Applied Physics Letters 99(16): 162904.

Koh SJA, Keplinger C, Li T, et al. (2011) Dielectric elastomer generators: how much energy can be converted? IEEE/ ASME Transactions on Mechatronics 16(1): 33-41.

McKay T, O’Brien B, Calius E, et al. (2010) An integrated, self-priming dielectric elastomer generator. Applied Physics Letters 97(6): 062911-1-062911-2.
Moretti G, Fontana M and Vertechy R (2013) Modeling and control of lozenge-shaped dielectric elastomer generators. In: Proceedings of ASME 2013 conference on smart materials, adaptive structures and intelligent systems, Snowbird, UT, 16-18 September, paper no. SMASIS2013-3258. New York: ASME.

Moretti G, Forehand D, Vertechy R, et al. (2014) Modeling of an oscillating wave surge converter with dielectric elastomer power take-off. In: Proceedings of 33rd international conference on offshore mechanics and arctic engineering, San Francisco, CA, 8-13 June, paper no. OMAE201423559. New York: ASME.

Pelrine R, Kornbluh R, Eckerle J, et al. (2001) Dielectric elastomers: generator mode fundamentals and applications. In: Proceedings of SPIE 4329: smart structures and materials 2001: electroactive polymer actuators and devices, Newport Beach, CA, 4-8 March, pp. 148-156. Bellingham, WA: SPIE.

Rosati Papini GP, Vertechy R and Fontana M (2013) Dynamic model of dielectric elastomer diaphragm generators for oscillating water column wave energy converters. In: Proceedings of ASME 2013 conference on smart materials, adaptive structures and intelligent systems, Snowbird, UT, 16-18 September, paper no. SMASIS2013-3255. New York: ASME.

Rosset S and Shea HR (2013) Flexible and stretchable electrodes for dielectric elastomer actuators. Applied Physics A: Materials Science \& Processing 110(2): 281-307.

Steinmann P, Hossain M and Possart G (2012) Hyperelastic models for rubber-like materials: consistent tangent operators and suitability for Treloar's data. Archive of Applied Mechanics 82(9): 1183-1217.

Tröls A, Kogler A, Baumgartner R, et al. (2013) Stretch dependence of the electrical breakdown strength and dielectric constant of dielectric elastomers. Smart Materials and Structures 22(10): 104012-104015.

Vertechy R, Berselli G, Parenti Castelli V, et al. (2010) Optimal design of lozenge-shaped dielectric elastomer linear actuators: mathematical procedure and experimental validation. Journal of Intelligent Material Systems and Structures 21(5): 503-515.

Vertechy R, Fontana M, Rosati Papini GP, et al. (2014a) Intank tests of a dielectric elastomer generator for wave energy harvesting. In: Proceedings of SPIE 9056: electroactive polymer actuators and devices (EAPAD), San Diego, CA, 9-13 March, pp. 90561G-1-90561G-11. Bellingham, WA: SPIE.

Vertechy R, Fontana M, Stiubianu G, et al. (2014b) Openaccess dielectric elastomer material database. In: Proceedings of SPIE 9056: electroactive polymer actuators and devices (EAPAD), San Diego, CA, 9-13 March, pp. 90561 R-1-90561 R-13. Bellingham, WA: SPIE.

Wang H, Zhu Y, Wang L, et al. (2012) Experimental investigation on energy conversion for dielectric electroactive polymer generator. Journal of Intelligent Material Systems and Structures 23(8): 885-895.

Zhu Y, Wang H, Zhao D, et al. (2011) Energy conversion analysis and performance research on a cone-type dielectric electroactive polymer generator. Smart Materials and Structures 20(11): 115022. 